\title{
Intrauterine contraceptive device migration to the urinary bladder
}

\author{
Urmila G. Gavali, Mayuri D. Pawar*, Gautam S. Aher, Suhas S. Shinde
}

Department of Obstetrics and Gynaecology, DVVPF's Medical College and Hospital, Ahmednagar, Maharashtra, India

Received: 13 August 2021

Accepted: 06 September 2021

\section{*Correspondence:}

Dr. Mayuri D. Pawar,

E-mail: mayuripawar43@gmail.com

Copyright: (c) the author(s), publisher and licensee Medip Academy. This is an open-access article distributed under the terms of the Creative Commons Attribution Non-Commercial License, which permits unrestricted non-commercial use, distribution, and reproduction in any medium, provided the original work is properly cited.

\begin{abstract}
Intravesical migration represent as uncommon complication of intrauterine device insertion. We present a case of an IUD that migrated to the urinary bladder and impacted in urinary bladder wall, causing significant urinary symptoms. A 44 years old woman presented with menorrhagia, lower abdominal pain and urinary symptoms since past 1 year. She reported an IUD insertion 10 years back. Imaging detected the presence of IUD in the urinary bladder wall with large fibroid in uterus. Under anaesthesia, total abdominal hysterectomy with bilateral salphingoophorectomy is performed and IUD was removed out of the bladder without any complications. In her follow-up evaluation after 2 week, she had no urinary symptoms. A regular follow-up of IUD for visible thread would help in earlier detection of misplaced IUD and prevent the complications such as intravesical migration.
\end{abstract}

Keywords: Intrauterine device, intrauterine device migration, cystostomy, urinary bladder

\section{INTRODUCTION}

Intrauterine device (IUD) is the most widely used reversible way of contraception worldwide. ${ }^{1}$ However, a vast variety of complications associated with IUD use has been encountered, ranging from discomfort after device placement to expulsion, PID, severe allergic reactions, perforations , migration and secondary calculi formation. ${ }^{2}$ Migrated intravesical IUD may present with no symptoms to non-specific urinary complaints, lower abdominal pain and even sexual dysfunction has been reported. ${ }^{3}$ The ultrasonography (USG) is the diagnostic modality of choice when searching for missing IUD because of its widespread availability, low cost, lack of ionizing radiation and minimally invasive nature. However CT has been employed for better localization and determining the relation of IUD with surrounding structures. Due to chronic inflammation produced by IUD, the need for necessary removal on diagnosis has to be emphasized. ${ }^{4,5}$ The preferred modality of management for removal of intravesical IUD is cystoscopic removal, however open cystectomy done in difficult and impacted cases.

\section{CASE REPORT}

A 44 years old married woman with previous 3 vaginal deliveries and 1 abortion with tubal ligation done 9 years back, farmer by occupation presented to the outpatient clinic complaining pain in lower abdominal pain, burning micturation and intermittent PV bleeding since 1 year. She has been taking symptomatic treatment for her urinary complaints, but due to her recurrent episodes of UTI she came to our institute for management. History of interval IUD insertion was elicited 6 months after abortion which was 11 years ago at private hospital which on follow ups was documented as lost IUD thread on per speculum examination and on USG no CuT was found in situ. However no further investigations were carried out in this regard. At the time of presentation, there was only suprapubic tenderness on abdominal examination. Per speculum examination did not reveal any IUD thread and rest of the examination was normal. Uterus were 8-10 weeks and adnexa were found normal on bimanual examination. The patient was advised ultrasound and urine for routine examination and culture sensitivity also performed. Ultrasound revealed a linear 
echogenic foreign body is seen in the bladder approximately measuring $36 \times 28 \mathrm{~mm}$. CT scan also revealed $37 \times 57 \mathrm{~mm}$ sized ' $\mathrm{T}$ ' shaped hyperdense lesion seen impacted along left lateral wall of urinary bladder wall resembling misplaced/migrated IUD and 50×45 mm moderately enhancing fibroid seen in myometrium of uterine fundus.

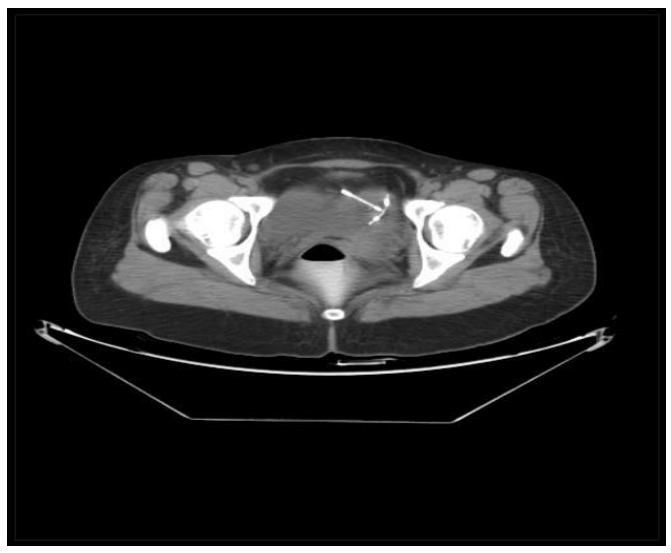

Figure 1: CT scan image showing $\mathrm{CuT}$ in bladder.

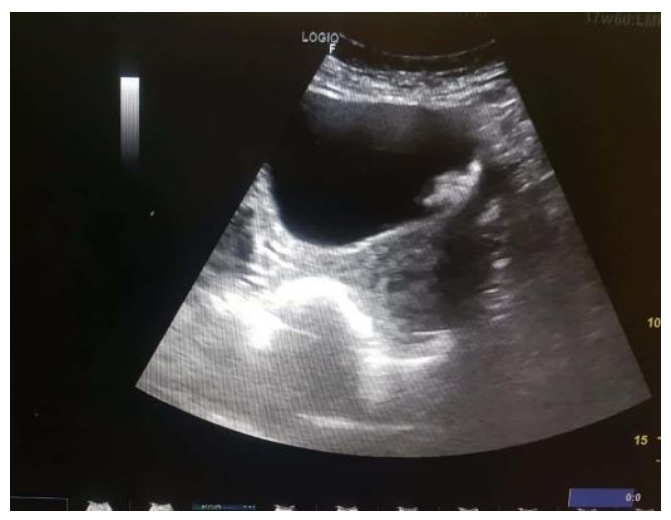

Figure 2: USG showing CuT in bladder.

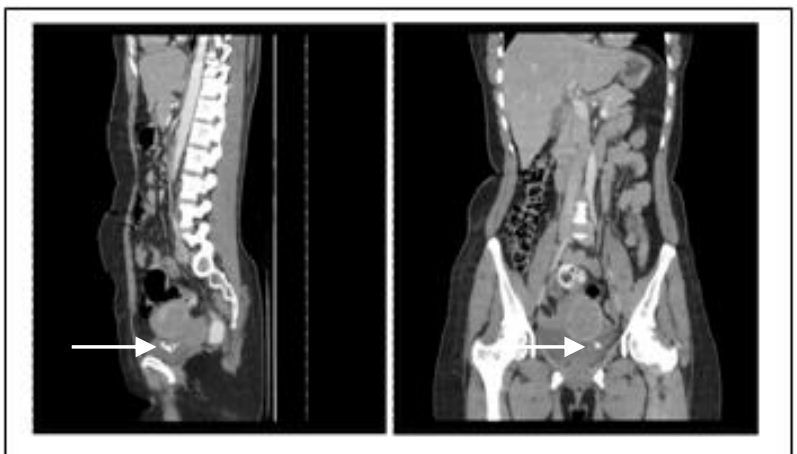

Figure 3: A) Coronal and B) Sagital sections of CT scan (abdo+pelvis) showing foreign body in bladder.

Her urine routine showed 10-12 CBC/hpf and culture was sterile. Under spinal anaesthesia cystostomy was done to remove IUD with total abdominal hysterectomy with bilateral salphingo-ophorectomy . Tip of arm of CuT would be palpated in left lateral wall of bladder. To access the $\mathrm{CuT}$ adhesions bladder mobilized. Evidence of omental and bowel adhsions to the left lateral wall of bladder with deeply impacted $\mathrm{CuT}$ into bladder wall. $\mathrm{CuT}$ arch steadied with babcocks forceps and dissected off bladder wall. Bladder defect sutured in 3 layers with vicryl 3-0. No bleeding was observed from the site of removal. Abdominal drain was left in situ and antibiotic was given postoperatively. The Foleys catheter was removed after 14 days and the patient was discharged. After 2 week following visit no urinary complaints were noted.

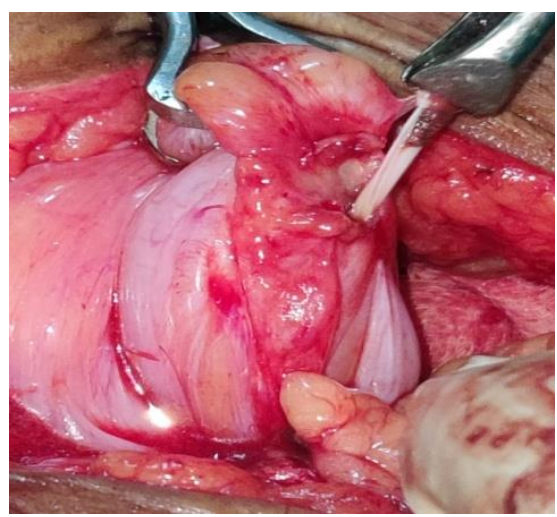

Figure 4: Intra-operative picture during removal of CuT.

\section{DISCUSSION}

IUD is a common contraceptive method for women of childbearing age. It is safe, effective, and easy to insert. Nevertheless, some complications may occur and include migration and embedding into the urinary bladder wall. ${ }^{5}$ The pathogenesis of uterine perforation by an IUD may occur by one of two mechanisms. The first is perforation at the time of IUD insertion, especially when associated with severe abdominal pain. The second proposed mechanism of perforation is by a gradual pressure necrosis of the uterine wall by the IUD (likely at its lead point) with eventual migration out of the uterus. $^{6}$ Migration and perforation may or may not be facilitated by uterine contractions. With complete perforation the IUD may be free in the peritoneal cavity or embedded in nearby structures or organs. Approximately $80 \%$ of uterine perforations are free in the peritoneal cavity. ${ }^{7}$ However, migration into adjacent organs with subsequent severe complications has been reported. IUD migration into adjacent organs has lead to bowel obstruction and perforation, peritonitis, appendicitis, vesical calculus formation, obstructive nephropathy, fistula formation, menouria, and intraperitoneal adhesions leading to infertility. ${ }^{8-10}$ Two types of uterine perforation have been characterized. Partial perforation is described as perforation that may advance through all the layers of the uterus, but some part of the IUD is retained within the uterine cavity or wall. An IUD may also be described as "embedded" to varying degrees within the uterine wall. Complete perforation occurs when all the layers of the 
uterine wall have been crossed, including the endometrium, myometrium, and serosa. ${ }^{6}$ Whatever the reasons may be, migrated IUDs that are symptomatic, and all copper IUDs (due to inflammation they produce) that have perforated the uterine wall, must be removed. ${ }^{1,11}$ Migrated IUDs can be removed either endoscopically, laparoscopically or with open surgery, depending on specific findings and patient's general condition.

In this case, the patient was asymptomatic in the first month after insertion, suggesting a migratory uterine perforation. The patient's initial episode of abdominal pain quickly resolved, possibly after the IUD had perforated and migrated out of the uterus. However, given that uterine perforation is often silent, it is difficult to say when the perforation occurred. The development of urinary symptoms few years after IUD insertion may be secondary to either the entrance of the IUD into the bladder or the development of a large calculus around the IUD in the bladder, subsequently causing irritative symptoms and burning micturation from contact with the bladder mucosa. In the reported case, urinary complaints in the form of burning micturation were the main symptoms that she developed few years following the insertion of the device. Urinary tract symptoms usually dominate the clinical presentation of a female patient with an IUD migrated to the bladder. The International Planned Parenthood Federation has recommended that all perforated IUDs be immediately removed given the rare but potentially catastrophic consequences. ${ }^{12}$ This topic has been debated; however, it is generally agreed that any IUD causing urinary symptoms should be removed promptly. $^{13}$

\section{CONCLUSION}

A regular follow-up of IUD for visible thread would help in earlier detection of misplaced IUD and prevent the complications such as intravesical migration. Proper training of paramedical staff is mandatory in developing countries to provide safe and better family planning services. In the reported case, lower abdominal pain and lower urinary tract symptoms were the leading complaints of an intravesical migration of an IUD.

Funding: No funding sources Conflict of interest: None declared

Ethical approval: Not required

\section{REFERENCES}

1. El-Hefnawy AS, El-Nahas AR, Osman Y, Bazeed MA. Urinary complications of migrated intrauterine contraceptive device. International Urogynecol J. 2008;19(2):241-5.
2. Tuncay YA, Tuncay E, Güzin K, Öztürk D, Omurcan C, Yücel N. Transuterine migration as a complication of intrauterine contraceptive devices: six case reports. Eur J Contracept Reprod Health Care. 2004;9(3):194200.

3. Dimitropolous K, Shripas K, et al. Intraut rie device migration to urinary bladder causing sexual dysfunction.a case report. Hippokratia. 2016;20(1): 70-2.

4. Wilson M, Whyte-Evans J. Use of volume imaging in the evaluation of intrauterine contraceptive devices. J Diag Med Sonograph. 2009;25(1):38-43.

5. Markovitch O, Klein Z, Gidoni Y, Holzinger M, Beyth Y. Extrauterine mislocated IUD: is surgical removal mandatory?. Contraception. 2002;66(2):1058.

6. Zakin, DS, Rosenblatt R. Complete and partial uterine perforation and embedding following insertion of intrauterine devices. II Diagnostic methods, prevention and management. Obstetrical Gynecol Surv. 1981;36(8):401-17.

7. Caspi B, Rabinerson D, Appelman Z, Kaplan B. Penetration of the bladder by a perforating intrauterine contraceptive device: a sonographic diagnosis. Ultrasound Obstet Gynecol. 1996;7(6):45860.

8. Rafique MR, Khan N, Haque TU. An unusual cause of vesical stone: a migrant intrauterine device. Eur J Contracept Reprod Health Care. 2003; 8:170-2.

9. Timonen H, Kurpa K. IUD perforation leading to obstructive nephropathy necessitating nephrectomy: a rare complication. Adv Contracept. 1987;3(1):71-5.

10. Schwartzwald DM, Tancer ML, Gomez-Leon G, Kim H. Vesicouterine fistula with menouria: a complication from an ntrauterine contraceptive device. J Urol. 1986;136(5):1066-7.

11. Nouira Y, Rakrouki S, Gargouri M, Fitouri Z, Horchani A. Intravesical migration of an intrauterine contraceptive device complicated by bladder stone: a report of six cases. Int Urogynecol J Pelvic Floor Dysfunct. 2007; 18:575-8.

12. Singh MM. IUDs and transmigration putting an old concern to rest. Int Planned Parenthood Fed Med Bull. 1995;29(1):2-3.

13. Kiilholma P, Makinene J, Vuori J. Bladder perforation: uncommon complications with a misplaced IUD. Contracept. 1989;5(1);47-9.

Cite this article as: Gavali UG, Pawar MD, Aher GS, Shinde SS. Intrauterine contraceptive device migration to the urinary bladder. Int J Reprod Contracept Obstet Gynecol 2021;10:4558-60. 